\title{
EL TRIUNFO DE TRUMPY SU IMPACTO SOBRE LAS RELACIONES ENTRE CHINA Y ESTADOS UNIDOS
}

Fabricio Antonio Fonseca Fernández

Universidad Nacional Chengchi

\section{INTRODUCCIÓN}

Entre los estudiosos de la ciencia política, particularmente entre los seguidores del institucionalismo, el inesperado triunfo electoral de Donald Trump será visto en retrospectiva como una coyuntura crítica, al haber presentado diversos retos para las instituciones estadounidenses e internacionales, que puede conducir a un ajuste importante de las mismas. El primer año de gobierno de Trump quedó marcado por su insistencia en desmontar el legado de su antecesor, Barack Obama, bajo la premisa de corregir los supuestos múltiples errores cometidos por la administración del demócrata, tanto dentro como fuera del país, con un profundo énfasis en la idea de "Estados Unidos primero". La política exterior estadounidense ha resentido dicho cambio, incluidas sus relaciones con la región Asia-Pacífico. Al cumplir con su promesa de campaña de retirar a Estados Unidos del Acuerdo de Asociación Transpacífico (TPP) en sus primeros días de gobierno, Trump intentó ser percibido como un defensor de los empleos industriales en su país, aunque en realidad el gobierno estadounidense continuó buscando otras formas de mantener e incrementar su influencia en esa parte del mundo.

Igualmente, la relación de Estados Unidos con la República Popular China (RPCh) ha entrado en una nueva fase, luego de una creciente retórica agresiva por parte de Trump en sus meses como candidato y una marcada posición de ambigüedad estratégica durante su 
primer año como presidente. La apariencia de parálisis y caos concurrente en Washington durante 2017 ha contrastado con la imagen de fortaleza del liderazgo de Xi Jinping en Beijing, particularmente tras los cambios anunciados durante el XIX Congreso Nacional del Partido Comunista Chino (PCCh). Con un gobierno estadounidense preocupado por revertir la reconversión industrial en el país, y por lo tanto poniendo en riesgo su apoyo a instituciones internacionales asociadas con la globalización y el libre comercio, y un gobierno chino que se promueve a sí mismo como un vocal defensor de dichos temas, y que se ha embarcado en la construcción de nuevas instituciones multilaterales y regionales, la relación bilateral más importante del sistema internacional ingresó en nuevos terrenos en meses pasados.

El presente capítulo ofrece un repaso de los principales tópicos que marcaron las relaciones sino-estadounidenses durante 2017, enfatizando los cambios promovidos por el lado norteamericano, las dificultades encontradas en el camino, así como las correspondientes reacciones de la contraparte china. El análisis refleja el alto grado de interdependencia económica que sigue prevaleciendo entre ambos países, así como un intento por parte de Washington de encontrar nuevos puntos de presión sobre Beijing, con la finalidad de obtener mayores concesiones económicas que puedan ser vendidas como importantes triunfos ante el electorado estadounidense.

Los temas de la política estadounidense de "una sola China" y las relaciones entre ambos lados del estrecho de Taiwán, así como el programa de armas nucleares de Corea del Norte, y en menor medida las disputas territoriales en el Mar del Sur de China, han sido vistos por la administración Trump como importantes asuntos que pueden ser vinculados con temas económicos y comerciales, con el fin de llevar la relación bilateral a un nuevo nivel, con un intercambio más equilibrado. Las siguientes secciones están dedicadas a cada uno de esos temas, incluidas también las implicaciones que los cambios promovidos por Washington pueden tener sobre otros actores de la región. Como los dos principales socios comerciales de México y de la gran mayoría de países en América Latina, es crucial entender el desarrollo de los vínculos sino-estadounidenses por los efectos que cualquier cambio inesperado puede tener en nuestras economías. 


\section{LA POLÍTICA DE "UNA SOLA CHINA"}

\section{COMO PUNTO DE PRESIÓN}

El 2 de diciembre de 2016 se dio a conocer una conversación telefónica sostenida entre Donald Trump y Tsai Ing-wen (Cai Yingwen), presidenta del gobierno de la República de China en Taiwán. Trump hizo uso de sus redes sociales para agradecer la llamada, y despertó la atención desmesurada de medios de comunicación y líderes políticos alrededor del mundo, por el significado del gesto. Por primera vez desde el rompimiento de relaciones diplomáticas de Estados Unidos con las autoridades de la isla, en enero de 1979, los líderes de los dos países entraban en contacto directo. ${ }^{1}$ Diversos analistas y estudiosos empezaron a debatir un posible cambio en la política estadounidense de "una sola China" bajo la presidencia de Trump, hacia una posición de mayor contacto con Taiwán, cuestionando el principio defendido por el gobierno de China continental, de que la isla forma parte inalienable de su territorio.

Como era de esperarse, Beijing condenó abiertamente el gesto, pero se abstuvo de apuntar directamente a Trump, y culpó entonces al gobierno taiwanés por su conducta temeraria. ${ }^{2}$ Lo anterior reflejó el creciente deterioro en las relaciones entre ambos lados del estrecho de Taiwán, tras el regreso del Partido Democrático Progresista (PDP) al poder ejecutivo de la isla, y su mayoría dentro del poder legislativo desde principios de 2016. Después de ocho años del gobierno de Ma Ying-jeou (Ma Yingjiu), del Partido Nacionalista Chino o Guomindang (GMD), la relación con China continental cambió de rumbo tras la llegada de Tsai. Bajo Ma hubo un acercamiento tácito con Beijing, mediante diversos

${ }^{1}$ Cabe recalcar que al momento de la llamada Donald Trump aún no asumía las funciones de presidente, ni su victoria había sido formalmente confirmada por el Colegio Electoral de Estados Unidos, por lo que contó con un mayor margen de maniobra para aceptar la comunicación de Tsai Ing-wen. No obstante, el gesto representó una primera crisis en las relaciones de la futura administración Trump con el gobierno de la RPCh. Anne Gearan, Philip Rucker y Simon Denyer, “Trump's Taiwan phone call was long planned, say people who were involved", The Washington Post, 4 de diciembre de 2016. Disponible en: <https:/www.washington post.com/politics/trumps-taiwan-phone-call-was-weeks-in-the-planning-say-people-who-were-involved/2016/12/ 04/f8be4b0c-ba4e-11e6-94ac-3d324840106c_story.html?utm_term=.4392d533a7a7>.

${ }^{2}$ Así lo hizo saber uno de los diarios de difusión internacional asociados con el Partido Comunista Chino. "Talk to Trump, punish Tsai Administration", Global Times, 4 de diciembre de 2016. Disponible en: <www.globaltimes.cn/content/1021908.shtml>. Véase también: Ben Blanchard, "China lodges protest after Trump call with Taiwan president", REUTERS, 3 de diciembre de 2016. Disponible en: $<$ https://www.reuters.com/ article/us-usa-trump-taiwan/china-lodges-protest-after-trump-call-with-taiwan-president-idUSKBN13R2NT>. 
acuerdos comerciales y una llamada "tregua diplomática" en la que ambos gobiernos se opusieron al establecimiento de relaciones diplomáticas con los aliados del otro.

Ante la negativa de la presidenta Tsai y el PDP de reconocer el llamado Consenso de 1992 como base de las relaciones entre ambos lados del estrecho, ${ }^{3}$ el gobierno chino comenzó a ejercer mecanismos de presión sobre su contraparte en la isla y rompió la tregua diplomática, como quedó demostrado en junio de 2017, con el caso de Panamá. Por lo tanto, la llamada telefónica a Trump fue considerada como una nueva provocación por parte de Taipéi, aunque los portavoces de los gobiernos de Xi y Obama procuraron asegurar que las relaciones sinoestadounidenses seguían siendo regidas por la política de "una sola China".

El presidente electo y algunos líderes republicanos justificaron la decisión de aceptar la llamada, al insistir en que se trató de una felicitación por parte de una presidenta elegida democráticamente, líder de un gobierno aliado cercano que adquiere miles de millones de dólares en armamento estadounidense. Los republicanos también contrastaron el hecho con el reciente establecimiento de relaciones diplomáticas con Cuba y la visita de Obama a la isla caribeña, controlada por un gobierno al que consideran un régimen dictatorial y enemigo histórico de Estados Unidos. En Taiwán, la llamada fue recibida con una mezcla de actitudes. A pesar de que el gobierno taiwanés intentó presentar el hecho como un acercamiento positivo con el que considera su aliado internacional más importante, y emocionó a algunos sectores favorables al reconocimiento oficial de un Taiwán soberano e independiente, la opinión pública en la isla se mostró dividida, y llegó incluso a sugerir el peligro de que Trump pudiera utilizar a Taiwán como una pieza de cambio en sus futuras relaciones con la $\mathrm{RPCh}^{4}$

${ }^{3}$ El Consenso de 1992 cobró fuerza durante la presidencia de Ma, refiriéndose a un acuerdo informal entre los dos lados del estrecho de Taiwán, donde ambos gobiernos reconocen la existencia de una sola China, pero admiten diferencias en la interpretación de la misma. El PDP ha rechazado constantemente dicho consenso, por considerarlo como un supuesto invento del GMD para mejorar las relaciones con Beijing, poniendo en peligro el futuro y la soberanía de la isla. Maeve Whelan-Wuest, "Former Taiwan president Ma on One China, the 1992 consensus, and Taiwan's future”, Order from Chaos, The Brookings Institution, 16 de marzo de 2017. Disponible en: <https:/www.brookings.edu/blog/order-from-chaos/2017/03/16/former-taiwan-president-maon-one-china-the-1992-consensus-and-taiwans-future/ $>$.

${ }^{4}$ En una pieza editorial escrita durante la campaña electoral, uno de los asesores económicos y de seguridad más radicales de Trump ya había rechazado la idea de utilizar a Taiwán como una pieza de negociación con el gobierno chino, lo que probablemente influyó después en el cambio de rumbo. Peter Navarro, “America Can't Dump Taiwan”, The National Interest, 19 de julio de 2016. Disponible en: <national interest.org/feature/america-cant-dump-taiwan-17040?page=show $>$. 
La incomodidad china creció cuando, el 9 de enero de 2017, durante una escala técnica en su visita a países con los que mantiene vínculos oficiales en Centroamérica, Tsai se reunió con el senador republicano Ted Cruz y el gobernador de Texas Greg Abbot en la ciudad de Houston. Varios temas fueron discutidos en la reunión, incluidos la venta de armamento y el aumento de contactos diplomáticos bilaterales. Cruz defendió su decisión y respondió a una nota diplomática por parte del consulado chino en Houston argumentando que China no puede tener derecho de veto sobre las reuniones oficiales mantenidas por el gobierno estadounidense. ${ }^{5}$ No obstante, no es difícil pensar que dicho encuentro influyó en la decisión posterior de Beijing de entablar relaciones diplomáticas con Panamá, que durante muchas décadas fue el aliado más importante de Taipéi en América Central.

La situación comenzó a cambiar luego de que Trump tomó posesión como presidente. Antes de entablar un diálogo formal con el gobierno chino, Trump se vio comprometido a aceptar oficialmente que su gobierno mantendría la política de "una sola China", observada por sus antecesores, y semanas después, en abril, admitió que no estaba entre sus planes recibir más llamadas telefónicas por parte de autoridades taiwanesas. Paradójicamente, dicha afirmación dio tranquilidad a varios sectores de Taiwán, los que después de la reunión Trump-Xi en Florida, a principios de abril, dejaron de percibir la amenaza de que Estados Unidos retirase su apoyo al gobierno isleño a cambio de mayores concesiones comerciales por parte de China continental. No obstante, el tema de Taiwán continuó empañando las relaciones sino-estadounidenses durante el primer año de mandato de Trump.

Después de que la administración de Jimmy Carter rompió relaciones diplomáticas con el gobierno de la isla y estableció vínculos formales con la RPCh, el Congreso estadounidense, tradicionalmente afín al gobierno nacionalista en Taipéi, aprobó la Ley de Relaciones de Taiwán (TRA, por sus siglas en inglés) en 1979, para conducir los vínculos con su antiguo aliado. Dicha ley obliga al gobierno estadounidense, entre otras cosas, a proveer de armas a Taiwán con el propósito de tener la suficiente capacidad de defensa, particularmente frente a cualquier intento de cambiar el statu quo entre ambas partes del estrecho por la fuerza. ${ }^{6} \mathrm{La}$

\footnotetext{
${ }^{5}$ Chris Horton, "Taiwan's President Meets With Ted Cruz in the U.S., and China Objects", The New York Times, 9 de enero de 2017. Disponible en: <https://www.nytimes.com/2017/01/09/world/asia/ taiwan-tedcruz-china.html>.

${ }^{6}$ Jacob K. Javits, "Congress and Foreign Relations: The Taiwan Relations Act”, Foreign Affairs, vol. 60, núm. 1 (otoño de 1981), pp. 54-62.
} 
venta de armas a la isla ha sido una constante fuente de fricciones en las relaciones sinoestadounidenses. Desde la época de Reagan, Washington se ha comprometido con Beijing a disminuir la cantidad de armas vendidas a Taiwán, así como ha dado garantías de vender únicamente equipo con fines de defensa, sin capacidad ofensiva. Los estadounidenses temen también que vender armamento tecnológicamente superior puede resultar contraproducente en el futuro, en el caso de una eventual reunificación de la isla con el continente. A pesar de ello, el 29 de junio de 2017, el gobierno de Trump confirmó su plan de vender armamento a Taipéi con un valor de \$1 420 millones de dólares, incluidos misiles y torpedos avanzados, así como asesoría técnica para la instalación de un sistema de radar de alerta temprana. ${ }^{7}$

El anuncio fue recibido con fuertes críticas por parte de Beijing, especialmente por tener lugar semanas después de la primera reunión entre los presidentes de ambos países, y justo en el día conmemorativo del vigésimo aniversario del regreso de Hong Kong a la soberanía china. De la misma forma, fue la primera vez en quince años que una administración estadounidense procedió a vender armas a la isla durante sus primeros meses en funciones. Las ventas de armamento a Taiwán durante los mandatos de George W. Bush y Barack Obama fueron disminuyendo en temporalidad y variedad, aunque siguieron provocando fuertes protestas por parte del gobierno chino, al considerarlo una injerencia directa en sus asuntos internos. Las autoridades estadounidenses respondieron, no obstante, afirmando que la venta de junio de 2017 había sido aprobada meses atrás, y que ésta no ha cambiado el apego de Washington a la política de "una sola China".

Por otra parte, la ambigüedad estratégica estadounidense volvió a ser patente en la segunda mitad del año, cuando el Comité de Relaciones Exteriores de la Cámara de Representantes presentó y aprobó un proyecto de ley para permitir un mayor intercambio de visitas entre funcionarios de Estados Unidos y Taiwán, seguido de una escala de Tsai en Hawai el 30 de octubre, en su visita a países aliados en el Pacífico Sur. ${ }^{8}$ Sin embargo, a pesar de la protesta de diplomáticos chinos, la iniciativa de ley tiene un largo camino por delante,

\footnotetext{
${ }^{7}$ Liu Zhen, "What arms is Taiwan actually getting for its US\$1.4 billion?", South China Morning Post, 4 de julio de 2017. Disponible en: <www.scmp.com/news/china/diplomacy-defence/article/2100793/ whatarms-taiwan-actually-getting-its-us14-billion>.

${ }^{8}$ CNA, "us House committee passes bill on Taiwan visits", Taipei Times, 14 de octubre de 2017. Disponible en: <www.taipeitimes.com/News/front/archives/2017/10/14/2003680301>; y Chung Li-hua, "Tsai visits Pearl Harbor memorial in Honolulu", Taipei Times, 30 de octubre de 2017. Disponible en: <www. taipeitimes.com/News/front/archives/2017/10/30/2003681305>.
} 
con menores posibilidades de ser aprobada por su contraparte en el Senado, así como por la difícil situación imperante en las relaciones entre los poderes legislativo y ejecutivo en Washington. Otra señal de la continua ambigüedad estratégica por parte de Estados Unidos fue la aprobación y publicación de la llamada Ley de Autorización de la Defensa Nacional (NDAA, por sus siglas en inglés). La ley contempla, entre otras cosas, la continuidad de la TRA y la autorización para visitas mutuas entre buques de guerra estadounidenses y taiwaneses. No obstante, a pesar de estas provisiones, que han sido bien vistas por Taipéi pero duramente cuestionadas por Beijing, expertos en Washington han sugerido que no está entre los planes de Trump enviar navíos de la Marina de visita al puerto de Kaohsiung (Gaoxiong). ${ }^{9}$

De esa forma, los meses posteriores a la elección y toma de protesta de Donald Trump como presidente quedaron marcados por su intento de utilizar a Taiwán como un punto de presión sobre el gobierno chino, aunque sin dar mucha continuidad a dicha estrategia. Ante los temores generalizados de un eventual abandono de la postura oficial ambigua respecto a la isla como parte de China, ambos lados del estrecho observaron un eventual retorno a una situación más familiar, con el gobierno estadounidense dando continuidad a los tres comunicados y la TRA, que son la base de sus relaciones con Taiwán y China continental. De la misma forma, a pesar de un nuevo gobierno del PDP en la isla, la presidenta Tsai se ha abstenido de recurrir a una retórica inflamatoria y provocativa, similar a la utilizada por el antecesor de su mismo partido, Chen Shui-bian (2000-2008).

El nuevo equilibrio de fuerzas en el estrecho de Taiwán, desfavorable para Taipéi, y el panorama cambiante en la política interna de la isla, con la popularidad de la presidenta continuamente a la baja, han forzado a Tsai a ser más cuidadosa en su política exterior y en sus relaciones con Beijing. De ahí que la estrategia de Trump para vincular un tema sensible para China con las cuestiones comerciales bilaterales, buscando obtener mayores ventajas, tuvo que cambiar de enfoque. A mediados de 2017, quedó claro que el programa de armamento nuclear de Corea del Norte sería uno de los nuevos puntos de presión.

\footnotetext{
${ }^{9}$ Matthew Strong, "Trump will not send U.S. Navy to Taiwan: former AIT officials", Taiwan News, 13 de diciembre de 2017. Disponible en: <https://www.taiwannews.com.tw/en/news/3319393>; y Jonathan Chin y Lu Yi-hsuan, "Taiwan thanks US over defense act", Taipei Times, 14 de diciembre de 2017. Disponible en: $<$ www.taipeitimes.com/News/front/archives/2017/12/14/2003683936>.
} 


\section{PERDIENDO LA PACIENCIA ESTRATÉGICA:}

\section{TRUMP Y EL PROGRAMA NUCLEAR NORCOREANO}

Después del encuentro entre Donald Trump y Xi Jinping en Mar-a-Lago, el líder estadounidense se mostró complaciente con la actitud de su homólogo chino y resaltó el compromiso asumido por este último respecto al asunto de Corea del Norte. De acuerdo con Trump, el gobierno chino acordó ejercer mayor presión sobre su vecino norcoreano, con el fin de contribuir al detenimiento y eventual desmantelamiento de su programa de armamento nuclear. El énfasis mostrado por el gobierno de Trump sobre la situación de la península coreana responde, en parte, al interés de distanciarse de las políticas seguidas por su antecesor. La administración de Obama se caracterizó por mostrar una actitud de "paciencia estratégica" hacia el gobierno de Corea del Norte y sus actos provocativos. ${ }^{10}$ Siguiendo un enfoque distinto debido a la naturaleza de cada caso, durante el segundo periodo de Obama, Washington buscó un acercamiento y logró cerrar un acuerdo con Teherán, a la vez que ignoró cualquier intento de diálogo y evitó una confrontación directa con Pyongyang.

Determinado a mostrar dichas políticas como un fracaso, con el fin de proyectar la imagen de un presidente que cumple sus promesas de campaña y seguir consolidando el apoyo entre su base electoral, Trump ha intentado poner fin al acuerdo con Irán y ha tomado una actitud más hostil hacia Corea del Norte. La percepción de amenaza norcoreana entre la sociedad estadounidense fue exaltada con el caso de Otto Warmbier, un estudiante estadounidense detenido y encarcelado en Pyongyang por más de un año. Su regreso a Estados Unidos, en estado de coma, y su eventual fallecimiento, hechos relacionados con una supuesta tortura sistemática por parte del gobierno norcoreano, ha sido utilizado por los medios estadounidenses y el gobierno como un ejemplo de la necesidad de endurecer la postura oficial frente al gobierno de Kim. ${ }^{11}$ De la misma manera, influenciado por sus asesores más conservadores, Trump ha intentado reforzar la narrativa de la amenaza del

\footnotetext{
${ }^{10}$ Jong Kun Choi, "The Perils of Strategic Patience with North Korea", The Washington Quarterly, vol. 38, núm. 4 (octubre de 2015), pp. 57-72.

${ }^{11}$ Gina Kolata, "Otto Warmbier Suffered Extensive Brain Damage, Coroner Confirms", The New York Times, 28 de septiembre de 2017. Disponible en: $<$ https://www.nytimes.com/2017/09/28/health/otto-warmbiernorth-korea-autopsy.html>.
} 
programa nuclear norcoreano como un problema en el que el gobierno chino necesita asumir una mayor responsabilidad.

En su lógica de encontrar puntos de presión para apalancar su posición negociadora, el gobierno de Trump ha mostrado su intención de endurecer su política comercial hacia Beijing si éste no logra contribuir al abandono de las ambiciones nucleares del régimen de Kim. Dicha situación ha puesto al gobierno chino en una posición similar a la de un equilibrista. Por una parte, los representantes de la RPCh han apoyado las consecutivas rondas de sanciones aprobadas por el Consejo de Seguridad de la ONU a lo largo de 2017, y por otra, anunciaron la prohibición de las importaciones norcoreanas de carbón y textiles, dos de los principales bienes en su comercio bilateral.

De igual manera, han impedido la exportación de derivados de petróleo e impuesto candados a instituciones bancarias chinas para realizar transacciones con empresas y negocios del vecino país. No obstante, el gobierno chino también se ha mostrado contrario a seguir endureciendo públicamente su retórica hacia Pyongyang, incluyendo una interrupción absoluta de sus exportaciones de petróleo a Norcorea. Asimismo, algunas de las sanciones chinas a empresas surcoreanas, como protesta por la instalación del sistema de lanzamiento de misiles THAAD, operado por Estados Unidos, han causado pérdidas considerables en dichas compañías y afectado sus vínculos con Seúl. De esa forma, una de las condiciones impuestas por Beijing para mejorar las relaciones con el gobierno surcoreano ha sido el cumplimiento de la promesa de este último para evitar la ampliación e instalación de nuevos sistemas THAAD en su territorio. De esa forma, un nuevo acercamiento con el gobierno de Corea del Sur, manifestado en la reciente visita de Moon Jae-in a Beijing, ha sido posible luego del anuncio hecho por Seúl sobre la no instalación de nuevos escudos antimisiles en su territorio. $^{12}$

El cambio en la postura estadounidense, con una continua subida de tono e intercambio de insultos entre Kim Jong-un y Donald Trump, motivó a Pyongyang a pisar el acelerador en

\footnotetext{
${ }^{12}$ Liu Zhen, "Why South Korea's promises on THAAD and a US-Japan alliance are so important to China", South China Morning Post, 6 de noviembre de 2017. Disponible en: <http://www scmp.com/news/china/diplomacy-defence/article/2118499/why-south-koreas-promises-thaad-and-us-japanalliance>.
} 
el desarrollo de su programa de armamento nuclear. ${ }^{13}$ En años anteriores, el gobierno norcoreano utilizaba fechas importantes en el calendario nacional para lanzar cohetes que supuestamente buscaban poner satélites en órbita. Para varios expertos en seguridad, los lanzamientos forman parte del programa de desarrollo de mísiles balísticos de alcance intercontinental, cuyo objetivo es tener la capacidad de transportar bombas nucleares a una mayor distancia, incluidas las costas del Pacífico en Estados Unidos. Tras la llegada de Trump a la Casa Blanca, las pruebas y lanzamientos norcoreanos han aumentado en temporalidad e intensidad, llegando incluso a hacer volar misiles sobre aguas territoriales de Japón, probar con éxito una bomba de hidrógeno, y amenazar con disparar bombas sobre Guam. ${ }^{14}$ Todo ello ha provocado que, al menos durante los primeros meses de su implementación, las sanciones económicas impuestas tengan un efecto contrario al esperado.

Conocedor de que una intervención militar estadounidense tendría repercusiones inmediatas sobre la capital surcoreana, lo que también es indeseable para el resto de países en la región, Kim ha proseguido con su programa de armamento nuclear, convencido de que es la única forma de alcanzar su objetivo último. La meta del gobierno de Corea del Norte es conseguir el reconocimiento oficial por parte de Estados Unidos, postergando no sólo la supervivencia del régimen, sino también reordenando el equilibrio de fuerzas en la península coreana. De la misma manera, y a pesar de un evidente alejamiento de Beijing desde el ascenso de Kim Jong-un, Pyongyang considera improbable un abandono por parte sus

\footnotetext{
${ }^{13}$ Susan B. Glasser, "Twitter Man vs. Rocket Man”, The Global Politico, Politico Magazine, 18 de septiembre de 2017. Disponible en: <www.politico.com/magazine/story/2017/09/18/donald-trump-northkorea-nuclear-weapons-kim-jong-un-215617>.

${ }^{14}$ Menos de un mes después de la toma de protesta de Donald Trump, el gobierno norcoreano realizó su primera prueba del año, lanzando un nuevo misil balístico, el Pukguksong-2, el día 12 de febrero de 2017. En los meses posteriores, y hasta el momento de escribir estas líneas, Pyongyang realizó pruebas en las siguientes fechas: 6 de marzo (lanzamiento de cuatro misiles sobre la zona económica exclusiva de Japón); 5 de abril (un misil balístico que explotó en el aire); 16 de abril (otro misil balístico que explotó luego de ser lanzado); 14 de mayo (misil balístico Hwasong-12, lanzado con éxito y considerado como de rango intermedio); 3 de julio (misil balístico Hwasong-14, considerado de alcance intercontinental); 28 de julio (reportado por otras potencias, con un radio de alcance mayor a los diez mil kilómetros); 25 de agosto (tres misiles balísticos de corto alcance); 28 de agosto (misil balístico Hwasong-12, sobrevoló Japón); 3 de septiembre (prueba nuclear, supuesta detonación exitosa de una bomba de hidrógeno); 15 de septiembre (misil balístico, probablemente Hwasong12, sobrevuela Japón), y 29 de noviembre (misil balístico, supuestamente Hwasong-15, de alcance intercontinental, sobre aguas japonesas). Un lanzamiento más fue esperado para la segunda mitad de diciembre, como conmemoración de la fecha de fallecimiento de Kim Jong-il. Por su parte, el 5 de agosto y el 11 de septiembre el Consejo de Seguridad de la ONU aprobó de forma unánime rondas de sanciones más rígidas contra el gobierno de Corea del Norte. Kelsey Davenport, "Chronology of U.S.-North Korean Nuclear and Missile Diplomacy", Fact Sheets \& Briefs, The Arms Control Association, 25 de septiembre de 2017. Disponible en: $<$ https://www.armscontrol.org/factsheets/dprkchron\#1999>.
} 
vecinos chinos, al considerar las implicaciones negativas que ello conllevaría para la estabilidad futura de la RPCh, particularmente en las provincias del noreste del país.

Un rompimiento abierto entre chinos y norcoreanos es improbable, debido a la posibilidad de que ello sea aprovechado por los estadounidenses en contra de Beijing. Dicho escenario representaría una repetición del acercamiento sino-estadounidense durante la Guerra Fría, cuando el programa nuclear chino fue un punto de tensión que terminó por minar la alianza entre chinos y soviéticos, empujando el péndulo a favor de Washington. De ahí que la necesidad de maniobras equilibristas sea cada vez más urgente y necesaria para la diplomacia china.

Sin embargo, la situación actual del gobierno estadounidense, de aparente caos, aumenta las tensiones y la incertidumbre acerca de una solución militar al problema. La posición de dureza del presidente contrasta con la voluntad negociadora de su secretario de Estado Rex Tillerson, quien siguió hablando de la necesidad de restablecer el diálogo con su par norcoreano a lo largo de 2017. Lo que algunos analistas interpretan como una estrategia bien planeada, al estilo de "policía bueno y policía malo", ha sido señalada por otros, como el senador republicano Bob Corker, como una simple muestra de la falta de dirección en la Casa Blanca, que puede impactar negativamente sobre el futuro del liderazgo estadounidense en el mundo. ${ }^{15}$

Asimismo, los primeros meses de la presidencia de Trump se caracterizaron por una creciente influencia de asesores militares, incluido su jefe de gabinete y anterior secretario de Seguridad Territorial, John Kelly, que ha sido determinante en las relaciones de Estados Unidos con otros países de Asia-Pacífico. Los ejercicios militares conjuntos, entre Seúl y Washington, y los contactos frecuentes con líderes japoneses, están encaminados a demostrar la continuidad de los compromisos estadounidenses con esos países en materia de seguridad y difuminar los temores a un eventual abandono de la región. De hecho, en la víspera de la primera gira de trabajo de Donald Trump a los países de Asia oriental, a inicios de noviembre, el Pentágono envió un reporte al Congreso afirmando que la única forma de terminar con el

\footnotetext{
${ }^{15}$ Anthony Zurcher, "Four ways Bob Corker skewered Donald Trump", BBC News, 9 de octubre de 2017. Disponible en: <www.bbc.com/news/world-us-canada-41419190>.
} 
programa nuclear norcoreano era mediante una intervención militar terrestre. ${ }^{16}$ Con ello se busca mandar la señal de que todas las opciones, incluida la militar, continúan sobre la mesa.

Por otro lado, la llegada del nuevo presidente surcoreano a la Casa Azul, de signo progresista-liberal, con un discurso más conciliador hacia su vecino del norte, tampoco ha obstaculizado una mayor cooperación entre el nuevo gobierno y su par norteamericano. Las constantes pruebas militares de Pyongyang han complicado el discurso de Moon a favor de la negociación y lo han acercado a la línea dura de Washington, a pesar de su intento de distanciarse de la política intercoreana seguida por el gobierno de su antecesora, la depuesta presidenta Park Geun-hye. Igualmente, la buena relación personal entre Donald Trump y Shinzo Abe, demostrada desde la primera visita de este último al complejo Mar-a-Lago, ha servido para incrementar la percepción de una inminente necesidad de resolver el problema norcoreano. De esa forma, el programa de armamento nuclear de Corea del Norte ha sido visto como un importante elemento de presión sobre China por parte del gobierno estadounidense, trasladando el tema, en ocasiones, a la discusión de asuntos económicos y comerciales. Estos últimos han probado ser una constante preocupación por parte de la administración Trump.

\section{LOS TEMAS COMERCIALES}

\section{Y EL RELANZAMIENTO DEL DIÁLOGO BILATERAL}

La campaña electoral de 2016 para elegir presidente en Estados Unidos repitió escenas del pasado, con el candidato del partido opositor criticando a la administración en turno por no hacer lo suficiente para evitar que China continúe obteniendo ventajas económicas frente a su país. No obstante, en esta ocasión, la retórica de Donald Trump como candidato cobró un tono más agresivo que el de competidores en elecciones pasadas. Las frases utilizadas por Trump, con su característico estilo incendiario, identificaban a China como el causante de gran parte de los males económicos de Estados Unidos, usando el marcado déficit comercial,

\footnotetext{
16 "Joint Chiefs say invasion 'only way' to totally disarm N Korea”, BBC News, 5 de noviembre de 2017. Disponible en: <www.bbc.com/news/world-us-canada-41878123>.
} 
que sobrepasa ya los 300 mil millones de dólares, como el principal indicador de una relación en extremo desventajosa.

Apelando al electorado de zonas gravemente afectadas por una gradual desindustrialización, provocada en parte por los efectos de la globalización y el traslado de fábricas a China y otras partes del mundo, Trump prometió, durante su primer día en funciones, imponer un arancel de $45 \%$ sobre las importaciones provenientes del país asiático, así como etiquetar oficialmente a este último como manipulador del tipo de cambio, con las consecuentes medidas punitivas sobre el comercio bilateral.

El inesperado triunfo de Trump llevó al liderazgo chino a cuestionarse de qué forma proceder. La experiencia acumulada llevaba a aconsejar prudencia, notando cómo la retórica de los candidatos estadounidenses no conduce a acciones drásticas una vez que éstos toman posesión del cargo. Sin embargo, al no ser un político tradicional y haber obtenido una victoria por medio de una estrategia poco convencional, las dudas sobre la seriedad de las amenazas de Trump entre los funcionarios chinos crecían semana tras semana. Conscientes de la enorme interdependencia económica de ambas potencias, y de las implicaciones para la propia economía estadounidense derivadas de acciones negativas en contra de sus pares chinos, el equipo de Trump buscó otros puntos de presión en la agenda bilateral para lograr reformular la relación económica y llevarla a un escenario más aceptable para los norteamericanos. La llamada teléfonica con Tsai Ing-wen, que tomó por sorpresa al gobierno chino y cuestionó por vez primera el apego de Washington a su política de "una sola China", fue interpretada como parte de esta nueva estrategia.

En un primer acercamiento, e intentando mostrar sus inconformidades, el consejero de Estado chino Yang Jiechi se reunió con Trump para señalar sus intenciones de no dejarse intimidar por las amenazas del magnate. Con las tensiones bilaterales a la alza, manifestadas también en el incidente del derribo de un dron estadounidense y la negativa inicial de regresarlo, el gobierno chino decidió cambiar de estrategia, buscando un enfoque más conciliador mediante un acercamiento con los familiares de mayor influencia sobre Trump, en particular su hija Ivanka y el esposo de ésta, Jared Kushner, quien ha sido señalado por 
tener importantes negocios en China. ${ }^{17}$ La utilidad del nuevo enfoque quedó evidenciada durante la primera reunión entre Xi y Trump. Después de lograr que el nuevo presidente estadounidense manifestara su compromiso de dar continuidad a la política de "una sola China", justo antes de la primera llamada telefónica entre ambos mandatarios, a inicios de febrero, y luego de la visita a Beijing del secretario de Estado Rex Tillerson, en la que reafirmó las intenciones estadounidenses de cooperar con China sobre una base de respeto y ganancias mutuas, el encuentro en Mar-a-Lago fue presentado como una oportunidad para motivar al gobierno chino a ofrecer mayores concesiones comerciales a cambio.

La reunión en Florida, alejada de las formalidades de Washington, sirvió para crear una relación más personal entre ambos líderes. De igual forma, los temas económicos y de Corea de Norte fueron confirmados como los más importantes en la agenda bilateral. En ese marco, la transformación del Diálogo Estratégico y Económico, a su vez heredero del Diálogo Económico Estratégico creado en 2006, en el nuevo Diálogo Económico Integral (Comprehensive Economic Dialogue o CED), fue celebrado como un paso hacia una reformulación de los principales aspectos económicos de las relaciones sinoestadounidenses. $^{18}$

De la misma manera, el anuncio de un plan bilateral de cien días, para la negociación de puntos económicos urgentes, buscó demostrar la intención del gobierno chino de conducir la relación por un camino constructivo. La reanudación del acceso al mercado chino de la carne de res estadounidense y una entrada limitada a ciertos sectores financieros de China fueron anunciadas por la administración de Trump como importantes logros en su relación con la RPCh. Dichas medidas fueron consecuentes con la necesidad del presidente

\footnotetext{
${ }^{17}$ La disposición de los familiares del presidente estadounidense para buscar una relación constructiva con China quedó de manifiesto con la asistencia de Ivanka Trump a las celebraciones oficiales del año nuevo chino en la embajada china en Washington, así como en las tradicionales canciones chinas interpretadas por la pequeña nieta de Donald Trump, Arabella Kushner, totalmente en mandarín, durante la visita de Xi Jinping y Peng Liyuan al complejo Mar-a-Lago en abril, y en un video que fue obsequiado a Xi durante la visita de Trump a Beijing en noviembre. Sobre el cambio de actitud del liderazgo chino hacia Donald Trump, veáse Eric Geller y Doug Palmer, "Is Trump backing down on China?", Politico Magazine, 7 de abril de 2017. Disponible en: $<$ http://www.politico.com/agenda/story/2017/04/trump-back-down-china-xi-visit-000402>.

${ }^{18}$ Para más información sobre el origen y la evolución de dichos mecanismos bilaterales de negociación véase Fabricio A. Fonseca Fernández, "Las relaciones de China con Estados Unidos en la era de la interdependencia económica y la evolución de sus percepciones", en Juan José Ramírez Bonilla y Francisco J. Haro Navejas (coords.), China y su entorno geopolítico: Políticas e instituciones de la integración regional, Ciudad de México, El Colegio de México, 2014.
} 
estadounidense de obtener ventajas de sus contrapartes, que puedan ser presumidas ante su base electoral como supuestos grandes triunfos, en su constante interés de presentarse como un negociador eficaz y conseguir su reelección en 2020.

Sin embargo, las expectativas para un reencauzamiento de la relación bilateral quedaron lejos de concretarse. En un año crucial para el fortalecimiento del liderazgo de Xi Jinping, con el desarrollo del XIX Congreso del PCCh a mediados de octubre, el presidente chino buscó mostrarse como un interlocutor responsable de cara a Donald Trump, pero evitó otorgar concesiones específicas, con el fin de no dar una imagen de sumisión o debilidad ante su contraparte estadounidense que pudiera ser usada en su contra por otros líderes o sectores del partido. ${ }^{19}$

De esa forma, la primera reunión del CED, llevada a cabo en Washington el día 19 de julio, encabezada por el secretario estadounidense del Tesoro Steven Mnuchin, el secretario estadounidense de Comercio Wilbur Ross y el vice-premier chino Wang Yang, terminó sin grandes anuncios. No obstante, la nueva dinámica del diálogo se diferenció de sus antecesores en aspectos importantes. En primer lugar, el número de funcionarios participantes disminuyó drásticamente, especialmente si consideramos que en las últimas ediciones durante la administración de Obama llegaron a viajar más de mil servidores públicos estadounidenses, de distintos niveles, a Beijing. ${ }^{20}$

Ambas partes también acordaron reducir la cantidad de temas a tratar, enfocándose exclusivamente en aquellos considerados prioritarios y en lineamientos generales, más que en casos específicos. Ejemplo de ello fue la falta de insistencia en un tema que fue recurrente durante los últimos años de Obama, relacionado con las supuestas actividades de espionaje chino para obtener secretos industriales, y en el que se lograron considerables avances. Aunque importante todavía, el tema no apareció esta vez en los titulares de los principales

${ }^{19}$ David Dollar y Ryan Hass, "Expect more process than progress at U.S.-China Comprehensive Economic Dialogue", Order from Chaos, The Brookings Institution, 18 de julio de 2017. Disponible en: $<$ https:/www.brookings.edu/blog/order-from-chaos/2017/07/18/expect-more-process-than-progress-at-u-schina-comprehensive-economic-dialogue/ $>$.

${ }^{20}$ Ello también se debió, en parte, a la inusual lentitud en la confirmación de varios puestos importantes en el gabinete y oficinas del ejecutivo federal en Washington. Scott Kennedy, "The Stalemate in U.S.-China Economic Talks: Turning a Corner or Going Around in Circles?", CSIS-Center for Strategic and International Studies, 3 de agosto de 2017. Disponible en: <https://www.csis.org/analysis/stalemate-us-china-economictalks-turning-corner-or-going-around-circles $>$. 
medios de comunicación, reflejando el cambio de rumbo en la nueva etapa del mecanismo bilateral.

Así pues, el primer encuentro del CED puede resumirse como una reunión en la que el gobierno estadounidense se dedicó a buscar mayores concesiones de los chinos, cuyo cumplimiento pudiese ser fácilmente verificable, enfatizando la necesidad de reducir el déficit comercial mediante una mayor apertura de la economía china en sectores clave para las empresas estadounidenses, a la vez que reafirmó las amenazas de recurrir a instrumentos comerciales unilaterales, particularmente en lo relacionado a la industria china del acero. ${ }^{21}$ El gobierno chino, por su parte, se limitó a tomar nota de las quejas estadounidenses y evitó llegar a compromisos concretos. No obstante, reconoció la preocupación estadounidense de reequilibrar el comercio bilateral, como una manera de profundizar el conocimiento y confianza mutuos, y adelantó la posibilidad de cambios en el mercado del arroz, para aceptar importaciones estadounidenses en un futuro próximo.

A pesar de una aparente frustración por parte de los funcionarios estadounidenses, ante una negativa china de conceder mayores privilegios económicos, el CED es también reflejo de la existente interdependencia de las dos mayores economías del mundo y demuestra que, a pesar de la retórica proteccionista de Donald Trump, el gobierno chino ha visto con buenos ojos el mantener este importante mecanismo de diálogo. Por otro lado, el presente escenario de interdependencia hace que cualquier medida unilateral por parte del gobierno estadounidense tenga también consecuencias catastróficas para su propia economía. No sólo se trata del gobierno chino como el mayor acreedor de Estados Unidos, por la enorme posesión de letras del Tesoro estadounidense, o de ser el país con las mayores reservas internacionales, sino que cualquier intento de imponer aranceles o medidas compensatorias sobre las exportaciones chinas puede ser respondido con medidas similares por parte de los chinos, afectando grandes porciones de las cadenas productivas y de valor norteamericanas, y de sectores estratégicos estadounidenses. Las presiones creadas por la interdependencia han provocado que los intentos de imponer medidas proteccionistas, o la estrategia de

${ }^{21}$ REUTERS, "US, China fail to agree on trade, casting doubt on other issues", $C N B C, 19$ de julio de 2017. Disponible en: <https://www.cnbc.com/2017/07/19/us-china-comprehensive-economic-dialoguedisagreement-over-how-to-reduce-trade-deficit-official-says.html $>$. 
vincular temas políticos y comerciales, como en el caso de Corea del Norte, hayan tenido que bajar de intensidad.

Ejemplo de ello son las medidas sobre el acero chino. Luego de haber obtenido la victoria en los llamados estados del cinturón del óxido, una zona caracterizada por la importancia de la industria acerera, el equipo de Trump procedió a encontrar la forma de presionar al gobierno chino en esa materia. Estados Unidos no es un gran importador de acero chino; no obstante, la industria estadounidense se ha visto afectada por la baja en los precios internacionales del acero, causada en parte por la creciente capacidad de producción y exportación de las compañías acereras chinas. Los grandes productores de otros países se han quejado de los subdisios y privilegios otorgados por el gobierno chino a las empresas productoras de ese país, que a la vez han hecho perder cuota de mercado a los conglomerados acereros de Estados Unidos.

En las distintas reuniones bilaterales, los funcionarios estadounidenses han traído el tema del acero como una importante fuente de preocupaciones de la administración Trump. Beijing ha tomado nota de la situación, y se ha propuesto seguir estudiando el tema, aunque sin comprometerse a dar grandes pasos inmediatos para disminuir la oferta de acero. La necesidad estadounidense de obtener mayores concesiones en este terreno ha movido a Washington a amenazar con recurrir a medidas unilaterales, específicamente la aplicación de la Sección 232 de la Ley de Expansión Comercial de 1962, para imponer cuotas a las importaciones de acero chino, al considerarlas como una amenaza a la seguridad nacional; o de la Sección 301 de la Ley de Intercambio de 1974, para investigar las prácticas comerciales chinas y su tratamiento a la inversión extranjera, con el fin de determinar si es necesaria la implementación de medidas compensatorias o aranceles comerciales. ${ }^{22}$

A pesar de las constantes advertencias, las consideraciones derivadas de la interdependencia y la aparente disposición china de aumentar las sanciones económicas sobre Pyongyang, llevaron a Trump a limitar el alcance de su amenaza, ordenando únicamente un estudio sobre la viabilidad de la aplicación de la Sección 301 para el caso del acero chino, así como para otras industrias semanas después. Lo mismo sucedió meses atrás. A pesar de haber prometido denunciar oficialmente al gobierno chino como manipulador de su moneda

\footnotetext{
${ }^{22}$ Scott Kennedy, "The Stalemate...", op. cit.
} 
en el primer día de su mandato, Trump se limitó a ordenar una investigación sobre los tipos de cambio en China y las implicaciones de tomar medidas punitivas, evitando ir más allá de eso. ${ }^{23}$ Una vez más, la presunta dureza de la nueva administración en Estados Unidos se ha topado con una realidad marcada por una interdependencia económica con China que puede tardar varios años en revertirse o ser disminuida sin acarrear graves daños colaterales.

\section{LA CONTINUIDAD DEL MAR DEL SuR DE CHINA COMO TEMA ESTRATÉGICO}

A pesar de caracterizarse por buscar un rompimiento con las medidas de su antecesor, existe un área de la política exterior hacia Asia-Pacífico a la que Donald Trump ha dado continuidad, la relacionada con el Mar del Sur de China. Después del fallo de un tribunal internacional en La Haya, refutando la validez de los reclamos históricos del gobierno chino sobre el espacio dentro de la llamada línea de nueve puntos o línea en forma de $\mathrm{U}$, el aparato militar estadounidense ha mantenido los llamados patrullajes sobre las zonas en disputa, con el supuesto objetivo de reforzar la libertad de navegación en esa parte del mundo. Por su parte, Beijing, a pesar de desconocer desde un principio la jurisdicción del tribunal sobre ese tema y no haber tomado parte en el proceso de investigación, ha buscado disminuir las tensiones, al tratar de revivir la negociación de un código de conducta con las otras partes reclamantes, por medio de la Asociación de Naciones del Sureste Asiático (ANSEA), y ha adoptado un perfil más bajo con respecto a la construcción de infraestructura sobre islas, islotes y arrecifes en el mar meridional. ${ }^{24}$ Igualmente, preocupado por las posibles repercusiones del fallo, particularmente las que pueden motivar a otros actores en la disputa a recurrir a instancias internacionales, el gobierno chino ha aprovechado el cambio de administración en Manila para modificar la actitud de esta última sobre el tema.

${ }^{23}$ Ana Swanson y Damian Paletta, "Trump says he will not label China currency manipulator, reversing campaign promise", The Washington Post, 12 de abril de 2017. Disponible en: <https://www. washingtonpost.com/news/wonk/wp/2017/04/12/trump-says-he-will-not-label-china-currency-manipulatorreversing-campaign-promise/?utm_term $=.15 \mathrm{dd} 2097 \mathrm{a} 7 \mathrm{ae}>$.

${ }^{24}$ Natalie Liu, "South China Sea Dispute Smolders as Trump Administration Weighs Approach", The Voice of America News, 22 de julio de 2017. Disponible en: <https://www.voanews.com/a/south-china-seadispute-smolders-trump-administration-weighs-approach/3954695.html>. 
La llegada al poder del controvertido presidente Rodrigo Duterte ha quedado marcada por un acercamiento con el gobierno chino y por una búsqueda para llegar a un acuerdo bilateral sobre la explotación de áreas y recursos en las zonas reclamadas. Diferente del camino seguido por el anterior mandatario, Benigno Aquino III, Duterte tomó distancia del gobierno de Obama durante sus primeros meses de gobierno, y tras la llegada de Trump ha aprovechado para obtener mayores concesiones por parte de Estados Unidos, incluida una decreciente crítica oficial sobre las graves violaciones a los derechos humanos y las acusaciones de asesinatos extrajudiciales cometidos durante la llamada guerra contra las drogas en Filipinas.

Consciente de la necesidad de mantener su influencia sobre la región y proyectar una imagen de confiabilidad ante sus aliados, la administración de Trump ha escuchado las voces en el ejército que hacen un llamado a buscar poner fin a los reclamos chinos sobre el Mar del Sur de China, principalmente a detener la construcción de lo que consideran islas artificiales que pueden ser utilizadas para proyectar el creciente poderío militar de la RPCh. Sin embargo, también existen voces que sugieren prudencia, notando que el fallo del tribunal representa un avance y que toca entonces conceder a la parte china un espacio para modificar el rumbo y desescalar tensiones sin hacerle perder cara. ${ }^{25}$

Por ello, el gobierno de Trump ha insistido en la importancia de resolver las disputas, aunque sin la misma intensidad y constancia demostrada durante los últimos años del gobierno de Obama. El tema fue traído nuevamente a discusión en las diferentes reuniones oficiales de alto nivel, incluidas la visita de Tillerson a Beijing, el encuentro Trump-Xi de Mar-a-Lago, y la visita oficial de Trump a la capital china; no obstante, no figuró como el tema principal de las conversaciones, y la parte china se limitó a tomar nota de la preocupación estadounidense sin ofrecer compromiso alguno en la materia. El paso de buques de guerra estadounidenses cerca de las islas disputadas y controladas por fuerzas chinas, un par de veces durante el verano de 2017, provocó molestia en Beijing, que calificó los actos de provocaciones militares y políticas. ${ }^{26}$

\footnotetext{
${ }^{25}$ Wendell Minnick, "Has the US lost the plot in South China Sea?", Shephard, 23 de junio de 2017. Disponible en: <https://www.shephardmedia.com/news/defence-notes/has-us-lost-plot-south-china-sea/>.

${ }^{26}$ Simon Denyer y Thomas Gibbons-Neff, "China vows to step up air, sea patrols after U.S. warship sails near disputed island", The Washington Post, 3 de julio de 2017. Disponible en: <https://www.washington post.com/world/china-accuses-us-of-serious-provocation-as-warship-sails-near-disputed-island/ 2017/07/02/ 9ce7b026-5f93-11e7-a6c7-f769fa1d5961_story.html?utm_term=.40d397ce0e07>.
} 
La continuidad de dichos patrullajes ha sido también interpretada como un punto de presión por parte de Estados Unidos para mover a la RPCh a una mayor cooperación, no sólo en lo relacionado con el Mar del Sur de China, sino también con otros temas considerados prioritarios, como el programa nuclear de Norcorea y los desequilibrios en el comercio bilateral. De ahí que se espere que los patrullajes continúen sobre una base constante en los próximos meses, y que la cooperación estadounidense con otras partes disputantes, principalmente Vietnam, siga en aumento. Ello ha quedado también en evidencia en la inclusión de los principales países disputantes en la gira de trabajo de Trump en la región, así como en su reunión con los países del ANSEA en Manila y su participación en la Cumbre de Asia Oriental, en la que ha manifestado su interés de dar continuidad al tema, lo que suele ser visto por el gobierno chino como un intento estadounidense de mantener su influencia en el área.

De hecho, la extensa gira realizada por Trump en la región en noviembre de 2017, bastante publicitada en medios estadounidenses y asiáticos, poco cambió el panorama de las relaciones bilaterales sino-estadounidenses y del papel jugado por Estados Unidos en Asia. El presidente estadounidense mostró su experiencia como hombre de negocios al elogiar a todos los líderes con los que se reunió, distanciándose de la práctica de tratar temas incómodos, como la protección de los derechos humanos, que era costumbre entre sus antecesores. Varios acuerdos bilaterales entre Estados Unidos y China fueron anunciados, pero ninguno con la suficiente trascendencia para corregir los desequilibrios comerciales o los patrones de inversión entre ambos países.

Por otro lado, el tema más recurrente durante la gira fue el continuo énfasis en la necesidad de resolver el problema norcoreano, tomándolo como un referente del compromiso estadounidense con la seguridad regional. De igual manera, la prensa prestó atención a las constantes alusiones a la región como Indo-Pacífico hechas por Trump, como señal del impulso estadounidense para incluir a los países del sur de Asia, específicamente India, así como ofrecer un mayor papel a Australia en la toma de decisiones con respecto al futuro de la región. Los llamados estadounidenses hacia la consecución de una región Indo-Pacífico libre y abierta pueden ser interpretados como una respuesta a la iniciativa china de la Franja y la Ruta de la Seda, que no incluye a Estados Unidos o a Japón, y a la que el gobierno indio 
se ha negado a respaldar, además de oponerse a algunos de esos proyectos por cuestiones de soberanía. ${ }^{27}$ No obstante, ante la retórica proteccionista característica de la nueva administración Trump, las señales contradictorias han movido a los gobiernos asiáticos a ser más cautelosos y no tomar partido, evitando quedar atrapados entre las dos potencias, a la vez que continúan obteniendo beneficios del crecimiento económico chino y de la presencia militar estadounidense como factor de estabilidad regional en materia de seguridad.

\section{CONSIDERACIONES PRELIMINARES}

Una de las referencias más recordadas y controvertidas durante el discurso de toma de posesión de Donald Trump fue el de "hecatombe estadounidense" (American carnage), entendida como el supuesto aprovechamiento y abuso de otros países sobre las bondades ofrecidas por un orden internacional liderado y sostenido por Estados Unidos, a costa de empleos y vidas de ciudadanos estadounidenses. El lema de campaña de Trump, acerca de recobrar la grandeza del país, apunta a una nueva fase de sensación generalizada de declive estadounidense, parecida a la provocada por el fracaso de la intervención en la Guerra de Vietnam, así como una vuelta a actitudes proteccionistas o aislacionistas, características de una cíclica política exterior estadounidense durante épocas pasadas. ${ }^{28}$

A la vez, la singular composición de la base electoral de Trump, particularmente la difícil alianza entre ultraconservadores religiosos, irreverentes nativistas y nacionalistas blancos, y halcones militares, entre otros, ha supuesto una falta de claridad sobre el rumbo del gobierno, incluida la política exterior, donde el mayor consenso parece ser el desmantelamiento del legado de Barack Obama. Todo ello ha llevado a un reordenamiento de prioridades en la relación de Estados Unidos con la región Asia-Pacífico, y particularmente con la RPCh, durante el primer año de Trump al frente del gobierno.

\footnotetext{
${ }^{27}$ Louis Nelson, "In Asia, Trump keeps talking about Indo-Pacific", Politico, 7 de noviembre de 2017. Disponible en: $<$ https://www.politico.com/story/2017/11/07/trump-asia-indo-pacific-244657>; PTI, "On OBOR, US backs India, says it crosses 'disputed' territory”, The Times of India, 4 de octubre de 2017. Disponible en: $<$ https://timesofindia.indiatimes.com/world/us/on-obor-us-backs-india-says-it-crosses-disputed-territory/articles how $/ 60934571 . \mathrm{cms}>$.

${ }^{28}$ Sobre la intermitente sensación de declive entre los estadounidenses y su afectación sobre la política exterior, véase Joseph S. Nye, Jr., "The Future of American Power: Dominance and Decline in Perspective", Foreign Affairs, vol. 89, núm. 6 (noviembre/diciembre de 2010), pp. 2-12.
} 
El libre comercio, anteriormente visto como una herramienta para mejorar las relaciones con otros países e incrementar la influencia estadounidense en el exterior, ha sido percibido por algunos asesores de Trump, principalmente antiglobalistas como el depuesto Steve Bannon y el radical Peter Navarro, como el culpable del relativo declive económico del país. Por ello, el ejecutivo ha convertido el déficit comercial con los países de Asia Oriental en una prioridad oficial, justificando así su retiro del TPP, su constante insistencia en renegociar el acuerdo comercial con Seúl, y la búsqueda de nuevos puntos de presión para hacer que China incremente sus importaciones de productos e inversiones estadounidenses.

Inicialmente, la política estadounidense de "una sola China" y su relación con Taiwán fueron pensados como una palanca sobre el gobierno chino. No obstante, después quedó claro que Corea del Norte y su programa de armas nucleares sería un tema en el que Washington encontraría un mayor apoyo dentro y fuera de Estados Unidos. La presión sobre Pyongyang y Beijing busca alejarse de la política de paciencia estratégica implementada por la administración de Obama, a la vez que mezcla temas políticos y comerciales con la finalidad de encontrar mayores elementos de presión y ventaja sobre la contraparte china.

El gobierno estadounidense ha buscado demostrar que mantiene su compromiso de defensa con los aliados regionales, pero ha insistido en que estos últimos necesitan incrementar sus gastos militares, así como ofrecer beneficios comerciales que puedan ser presentados por Trump como triunfos económicos ante su electorado. Para el caso de China, Washington ha intentado también obtener mayores concesiones comerciales y simplificar los mecanismos de diálogo económico bilateral implementados desde los años de George W. Bush, pero los resultados y beneficios obtenidos han sido menores a lo esperado.

En un año clave para el fortalecimiento del liderazgo de Xi Jinping en China, Trump y su equipo buscaron evitar una escalada de presiones directas sobre Beijing, aunque han mantenido parte de su retórica agresiva, en gran medida destinada a audiencias locales, como en el caso de la identificación de China como un responsable de la actual crisis de adicción a opioides en Estados Unidos por las supuestas exportaciones encubiertas de fentanilo, utilizado en la producción de drogas sintéticas. La identificación de China y Rusia como rivales de Estados Unidos en la recientemente publicada estrategia de seguridad, aunque llamando a la continua cooperación con ambos, pone de manifiesto un cambio en la 
conducción estadounidense de su política exterior, incluida su relación con el gigante asiático. Así, la constante interdependencia económica sino-estadounidense continúa obligando a ambas partes a negociar y disminuye las posibilidades de una guerra comercial con graves consecuencias para ambos países. Si bien la constante visión de ganancias absolutas y un juego de suma cero por parte de la administración Trump hace más difícil la cooperación con China, la idea de pérdidas aún mayores, derivadas de un repentino reordenamiento unilateral del comercio, ha forzado a Washington a mantener abierta la opción del diálogo con Beijing, a la vez que continuará la búsqueda de nuevos puntos de presión sobre una China que sigue incrementando sus capacidades materiales, su proyección internacional y su influencia sobre Asia-Pacífico y otras regiones del mundo.

\section{BIBLIOGRAFÍA}

BBC News, “Joint Chiefs say invasion 'only way' to totally disarm N Korea”, BBC News, 5 de noviembre de 2017. Disponible en: <www.bbc.com/news/world-us-canada-41878123>.

Blanchard, Ben, "China lodges protest after Trump call with Taiwan president”, REUTERS, 3 de diciembre de 2016. Disponible en: <https://www.reuters.com/article/us-usa-trumptaiwan/china-lodges-protest-after-trump-call-with-taiwan-president-idUSKBN 13R2NT>.

Chin, Jonathan y Lu Yi-hsuan, "Taiwan thanks US over defense act", Taipei Times, 14 de diciembre de 2017. Disponible en: <www.taipeitimes.com/News/front/archives/2017/ $12 / 14 / 2003683936>$.

CNA, "US House committee passes bill on Taiwan visits", Taipei Times, 14 de octubre de 2017. Disponible en: <www.taipeitimes.com/News/front/archives/2017/10/14/200368 $0301>$.

Davenport, Kelsey, "Chronology of U.S.-North Korean Nuclear and Missile Diplomacy", Fact Sheets \& Briefs, The Arms Control Association, 25 de septiembre de 2017. Disponible en: <https://www.armscontrol.org/factsheets/dprkchron\#1999>. 
Denyer, Simon y Thomas Gibbons-Neff, "China vows to step up air, sea patrols after U.S. warship sails near disputed island", The Washington Post, 3 de julio de 2017. Disponible en: $<$ https://www.washingtonpost.com/world/china-accuses-us-of-seriousprovocation-as-warship-sails-near-disputed-island/2017/07/02/9ce7b026-5f93-11e7a6c7-f769fa1d5961_story.html?utm_term $=.40 \mathrm{~d} 397 \mathrm{ce} 0 \mathrm{e} 07>$.

Dollar, David y Ryan Hass, "Expect more process than progress at U.S.-China Comprehensive Economic Dialogue", Order from Chaos, The Brookings Institution, 18 de julio de 2017. Disponible en: <https://www.brookings.edu/blog/order-fromchaos/2017/07/18/expect-more-process-than-progress-at-u-s-china-comprehensiveeconomic-dialogue/>.

Fonseca Fernández, Fabricio A., "Las relaciones de China con Estados Unidos en la era de la interdependencia económica y la evolución de sus percepciones”, en Juan José Ramírez Bonilla y Francisco J. Haro Navejas (coords.), China y su entorno geopolítico: Políticas e instituciones de la integración regional, Ciudad de México, El Colegio de México, 2014.

Gearan, Anne, Philip Rucker y Simon Denyer, “Trump's Taiwan phone call was long planned, say people who were involved", The Washington Post, 4 de diciembre de 2016. Disponible en: <https://www.washingtonpost.com/politics/trumps-taiwan-phonecall-was-weeks-in-the-planning-say-people-who-were-involved/2016/12/04/f8be4b0cba4e-11e6-94ac-3d324840106c_story.html?utm_term=.4392d533a7a7>.

Geller, Eric y Doug Palmer, “Is Trump backing down on China?”, Politico Magazine, 7 de abril de 2017. Disponible en: <http://www.politico.com/agenda/story/2017/04/trumpback-down-china-xi-visit-000402>.

Glasser, Susan B., “Twitter Man vs. Rocket Man”, The Global Politico, Politico Magazine, 18 de septiembre de 2017. Disponible en: <www.politico.com/magazine/story/2017/ 09/18/donald-trump-north-korea-nuclear-weapons-kim-jong-un-215617>.

Global Times, "Talk to Trump, punish Tsai Administration”, Global Times, 4 de diciembre de 2016. Disponible en: <www.globaltimes.cn/content/1021908.shtml>. 
Horton, Chris, "Taiwan's President Meets With Ted Cruz in the U.S., and China Objects", The New York Times, 9 de enero de 2017. Disponible en: <https://www.nytimes. com/2017/01/09/world/asia/ taiwan-ted-cruz-china.html>.

Javits, Jacob K., "Congress and Foreign Relations: The Taiwan Relations Act", Foreign Affairs, vol. 60, núm. 1 (otoño de 1981), pp. 54-62.

Kennedy, Scott, "The Stalemate in U.S.-China Economic Talks: Turning a Corner or Going Around in Circles?", CSIS-Center for Strategic and International Studies, 3 de agosto de 2017. Disponible en: <https://www.csis.org/analysis/stalemate-us-china-economictalks-turning-corner-or-going-around-circles $>$.

Kolata, Gina, "Otto Warmbier Suffered Extensive Brain Damage, Coroner Confirms", The New York Times, 28 de septiembre de 2017. Disponible en: <https://www.nytimes. com/2017/09/28/health/otto-warmbier-north-korea-autopsy.html>.

Kun Choi, Jong, "The Perils of Strategic Patience with North Korea", The Washington Quarterly, vol. 38, núm. 4 (octubre de 2015), pp. 57-72.

Li-hua, Chung, "Tsai visits Pearl Harbor memorial in Honolulu”, Taipei Times, 30 de octubre de 2017. Disponible en: <www.taipeitimes.com/News/front/archives/2017/10/30/2003 $681305>$.

Liu, Natalie, "South China Sea Dispute Smolders as Trump Administration Weighs Approach", The Voice of America News, 22 de julio de 2017. Disponible en: <https:// www.voanews.com/a/south-china-sea-dispute-smolders-trump-administration-weighsapproach/3954695.html>.

Minnick, Wendell, “Has the US lost the plot in South China Sea?”, Shephard, 23 de junio de 2017. Disponible en: <https://www.shephardmedia.com/news/defence-notes/has-uslost-plot-south-china-sea/>.

Navarro, Peter, “America Can’t Dump Taiwan”, The National Interest, 19 de julio de 2016. Disponible en: <nationalinterest.org/feature/america-cant-dump-taiwan-17040?page= show $>$. 
Nelson, Louis, "In Asia, Trump keeps talking about Indo-Pacific", Politico, 7 de noviembre de 2017. Disponible en: <https://www.politico.com/story/2017/11/07/trump-asia-indopacific-244657>

Nye, Joseph S. Jr., "The Future of American Power: Dominance and Decline in Perspective”, Foreign Affairs, vol. 89, núm. 6 (noviembre/diciembre de 2010), pp. 2-12.

PTI, “On OBOR, US backs India, says it crosses 'disputed' territory”, The Times of India, 4 de octubre de 2017. Disponible en: <https://timesofindia.indiatimes.com/world/us/onobor-us-backs-india-says-it-crosses-disputed-territory/articleshow/60934571.cms>.

REUTERS, "US, China fail to agree on trade, casting doubt on other issues", $C N B C, 19$ de julio de 2017. Disponible en: <https://www.cnbc.com/2017/07/19/us-china-comprehensiveeconomic-dialogue-disagreement-over-how-to-reduce-trade-deficit-official-says.html>.

Strong, Matthew, "Trump will not send U.S. Navy to Taiwan: former AIT officials", Taiwan News, 13 de diciembre de 2017. Disponible en: <https://www.taiwannews.com.tw/ en/news/3319393>.

Swanson, Ana y Damian Paletta, "Trump says he will not label China currency manipulator, reversing campaign promise", The Washington Post, 12 de abril de 2017. Disponible en: <https://www.washingtonpost.com/news/wonk/wp/2017/04/12/trump-says-he-willnot-label-china-currency-manipulator-reversing-campaign-promise/?utm_term $=.15$ dd2097a7ae>.

Whelan-Wuest, Maeve, "Former Taiwan president Ma on One China, the 1992 consensus, and Taiwan's future", Order from Chaos, The Brookings Institution, 16 de marzo de 2017. Disponible en: <https://www.brookings.edu/blog/order-from-chaos/2017/03/16/ former-taiwan-president-ma-on-one-china-the-1992-consensus-and-taiwans-future/>.

Zhen, Liu, "What arms is Taiwan actually getting for its US\$1.4 billion?", South China Morning Post, 4 de julio de 2017. Disponible en: <www.scmp.com/news/china/diplomacydefence/article/2100793/what-arms-taiwan-actually-getting-its-us14-billion>.

Zhen, Liu, "Why South Korea's promises on THAAD and a US-Japan alliance are so important to China", South China Morning Post, 6 de noviembre de 2017. Disponible en: 
$<$ http://www.scmp.com/news/china/diplomacy-defence/article/2118499/why-southkoreas-promises-thaad-and-us-japan-alliance>.

Zurcher, Anthony, "Four ways Bob Corker skewered Donald Trump", BBC News, 9 de octubre de 2017. Disponible en: <www.bbc.com/news/world-us-canada-41419190>. 